\title{
Estimating risk of exposure induced cancer death in patients undergoing computed tomography pulmonary angiography
}

\author{
H. Karimizarchi ${ }^{1}$ and A. Chaparian ${ }^{1,2, *}$ \\ ${ }^{1}$ Medical Physics Department, Shahid Sadoughi University of Medical Sciences, Yazd, Iran. \\ 2 Technology of Radiology Department, Isfahan University of Medical Sciences, Isfahan, Iran.
}

Received: 30 January 2016 / Accepted: 5 December 2016

\begin{abstract}
The purpose of the study is to estimate the risk of exposure-induced cancer death (REID) values for computed tomography pulmonary angiography (CTPA) examination. During the study period, 152 patients ( 77 women and 75 men) were referred for evaluation of suspected pulmonary embolism with CTPA examination. Organ doses and the effective dose were calculated by means of Monte Carlo simulations for each patient. The REID values were estimated for CTPA, using models developed in the Biological Effects of Ionizing Radiation VII - Phase 2 report. The REID values varied from 53 to 248 per million depending on the age and gender of patients. These values were considerably higher for females $(173 \pm 61$ per million) than males ( $82 \pm 26$ per million) and higher for younger than older patients. The REID values changed with age at exposure by a about factor of 3-5 between the ages of 18 year and 89 year. Results of this study will help the referring physicians to weigh medical benefits of CTPA in clinical practice against possible risks to justify each CTPA decision in order to reduce unnecessary examinations or use alternative imaging modalities with low risk.
\end{abstract}

Keywords: computed tomography / angiography / Monte Carlo simulations / radiation risk

\section{Introduction}

Pulmonary embolism (PE) is a considerable cause of mortality. It affects nearly 600,000 persons and may result in approximately 100,000 deaths per year in the United States (Surgeon General, 2008). An accurate procedure for imaging of the pulmonary arteries and diagnosis of PE is computed tomography pulmonary angiography (CTPA). However, the ionizing radiation doses delivered by $\mathrm{CT}$ are high and increase the rate of cancer induction and mortality risk (ICRP Publication 60, 1991; NRC, 2006; Preston et al., 2007). The International Commission for Radiological Protection (ICRP) recommends that examinations involving ionizing radiation should fulfill the two basic principles of justification of practice and optimization of protection (ICRP Publication 34, 1982; ICRP Publication 60, 1991; ICRP Publication 73, 1996). Justification is achieved by providing physicians with information about the potential health effects from each medical exposure based on an evaluation of the risk of any detrimental effects that can be weighed against the medical benefits. Also, results of some studies (Wells et al., 2001; Musset et al., 2002; Diederich, 2003; O'Neill et al., 2004, 2005; Costantino et al., 2008; He et al., 2012) have shown that only $9-35 \%$ of CTA scans ordered for suspected PE yield

\footnotetext{
* Corresponding author: ali_chaparian@yahoo.com
}

positive findings. Therefore awareness of the potential risks from a CTPA examination is necessary for taking decisions about performing the study in question.

Contrary to the computed tomography coronary angiography that has been studied in detail, few studies described radiation dose associated to CTPA. Heyer et al. (2007) calculated the effective dose for CTPA on the basis of dose length product (DLP) and volume CT dose index (CTDI $\left.\mathrm{VOL}_{\mathrm{VO}}\right)$. They compared 16-section multi detector CT at 100 and $120 \mathrm{kVp}$ for image quality and radiation dose. Woo et al. (2012) estimated the lifetime attributable risk of cancer mortality due to radiation from CTPA by calculating the estimated effective dose and using gender-specific polynomial equations derived from the Biological Effects of Ionizing Radiation VII (BEIR VII) report. They also calculated benefitto-risk ratios by dividing the mortality benefit of preventing a fatal PE by the mortality risk of a radiation-induced cancer. Yilmaz et al. (2013) evaluated the effectiveness of different tube voltages uses in CTPA in the diagnosis of PE. They also found the optimal tube voltage value and studied the possibility of obtaining appropriate imaging quality with minimal radiation dose.

To date, most studies focused on the calculation of effective dose for CTPA examination. Even in the study conducted by Woo et al. (2012), the risk estimation was also based on effective dose. However, the uncertainty in estimated values of the effective dose is about $\pm 40 \%$ for a reference 
patient of $70 \mathrm{~kg}$, since organ doses and tissue weighting factors used for computing effective doses are averaged over all ages and both genders in the general population. The uncertainty in risk of cancer may be a factor of three higher or lower for a reference patient, and will be more variable for an individual (Martin, 2007; McCollough et al., 2010). Since radiation risks and sensitivities for different tissues vary with gender and age at exposure (ICRP Publication 60, 1991; NRC, 2006), the assessment of cancer risk based on effective dose for an individual can lead to an underestimation for children of both genders by a factor of 1.5-4 and an overestimation for adults patients by a factor of 2.5 and 10, respectively (Martin, 2011; Balonov and Shrimpton, 2012). Therefore, the risk assessment should be based on organ dose estimates for the age and gender of the patients concerned. Several investigations (ICRP Publication 103, 2007; Martin, 2007; Brenner, 2008; McCollough et al., 2010; Huda and He, 2012; Pradhan et al., 2012) also suggested that age, gender, and tissue-specific risk coefficients are used instead of effective dose to estimate radiation risk. The purpose of this study was to determine organ doses and to estimate risk of exposure-induced cancer death (REID) values for CTPA examination.

\section{Materials and methods}

Between June 2014 and July 2015, all consecutive patients who were referred to the computed tomography department of Shohaday-e Kargar hospital (Yazd, Iran) for CTPA examination were considered in this study. All scans were obtained by using a 16-slice multidetector-row CT scanner (Somatom Emotion 16, Siemens AG, Munich, Germany) with spiral technique. Patients were scanned using tube current modulation (reference tube current-time product of $80 \mathrm{mAs}$ ), a tube voltage of $110 \mathrm{kV}$, gantry rotation speed of $0.6 \mathrm{~s}$, collimation of $16 \times 0.6 \mathrm{~mm}$, slice thickness of $0.6 \mathrm{~mm}$ and a pitch factor of 1.5. The effective diameter of patients, scan length, effective tube current-time product, and DLP values were recorded from the CT scanner console. The accuracy of the displayed CTDI and DLP values was verified by measuring doses in a Perspex phantom (diameter of $32 \mathrm{~cm}$ and length of $15 \mathrm{~cm}$ ) using a pencil probe solid state dosimeter (Unfors Mult-OMeter 601, Sweden) according to literature (Shope et al., 1981; IAEA, 2012). The discrepancy between measured and displayed CTDI values was $< \pm 10 \%$.

Effective dose $(E)$ is derived from the weighted sum of doses to organs by the equation: $E=\sum W_{T} \cdot H_{T}$, where $E$ is the product of the tissue weighting factor $\left(W_{T}\right)$, which represents the relative contribution of that organ or tissue to the overall risk, and the equivalent dose $H_{T}$.

Organ doses and effective dose were determined by the ImpactDose program (VAMP GmbH, Erlangen, Germany). Dose calculations in the ImpactDose are based on tabulated Monte Carlo calculation results achieved by the validated Monte Carlo program ImpactMC (Deak et al., 2008; Chen et al., 2012). The ImpactDose program was previously validated in several studies (Kalender et al., 1999; Deak et al., 2008; Chen et al., 2012) by dose measurements for different scan protocols. The dose distributions were simulated and compared with thermoluminescent dosimeters measurements in anthropomorphic phantoms of various sizes.
The calculated dose values were generally within $10 \%$ of measurements for all investigated conditions.

Two types of anthropomorphic phantoms are available for the patient model in the ImpactDose program. The user can either use the default voxel phantoms (a standard male and female voxel phantom according to ICRP 110 (2009)) by choosing "voxel phantom" or use the Oak Ridge National Laboratory (ORNL) phantom series (Cristy and Eckerman, 1987) to adapt the calculation to the individual patient's parameters by choosing "patient data". If "patient data" is chosen, a linear interpolation is performed between versions of the ORNL phantom series. This interpolation is performed using the "effective diameter" of the patient, which is the square root of the product of lateral (Lat) and anterior-posterior (AP) diameter. The user can either choose to enter the effective diameter directly or give "AP" and "Lat" diameters obtained from CT scans of each patient, which will then be converted to a corresponding effective diameter by the ImpactDose program. For default voxel phantoms, patient gender needs to be specified while the patient data model requires patient age, gender, and diameter (ImpactDose manual). Since it has been shown that organ doses strongly depend on patient size (Turner et al., 2011), in this study, we chose the ORNL phantom series option. The effect of effective diameters on radiation dose has been investigated in several studies (Turner et al., 2010; AAPM, 2011; Li et al., 2011; Sahbaee et al., 2014).

The ImpactDose can calculate effective dose by using both the earlier WT (ICRP 26 and 60) (ICRP Publication 26, 1977; ICRP Publication 60, 1991) and the new ones (ICRP 103) (ICRP Publication 103, 2007). In this investigation, organ doses were calculated in 28 organs and effective dose was obtained using the new tissue weighting factors of ICRP Publication 103 (2007).

The REID values were estimated for CTPA, using models developed in the BEIR VII - Phase 2 report (NRC, 2006). Organ doses calculated by the ImpactDose program were used for the assessment of the REID values. The PCXMC Monte Carlo code developed by STUK (Radiation and Nuclear Safety Authority in Finland) was used for the calculation of REID values. The PCXMC program is able to calculate organ doses, and effective doses of patients in $\mathrm{X}$-ray projection radiographies. Also, this program has the ability to estimate the risk of exposure-induced cancer from organ dose files of X-ray examinations simulated in the program and similarly from the organ dose files of arbitrary irradiation cases. Thus, for REID estimation of the CTPA examinations in this study, we manually changed the format and font of the organ dose files calculated by the ImpactDose to be compatible with the input files of the PCXMC. The risk of exposure-induced cancer assessment in PCXMC is performed according to the sex- and age-dependent risk model of the BEIR VII Committee. Risk models are available for leukemia, cancers in colon, stomach, lung, urinary bladder, prostate, uterus, ovaries, breast, liver, thyroid, and for all other solid cancers combined. For all cancer types, the BEIR VII had obtained absolute and relative risk models. Excess cancer risk from radiation is independent of the background cancer risk (i.e., cancers from other causes than radiation) in the absolute risk model, but in the relative risk model, the radiation risk is proportional to the background cancer risk. Both models were combined in the BEIR VII for 
Table 1. The characteristics of patients including number, age, effective diameter and length of the scanning area.

\begin{tabular}{|c|c|c|c|c|c|c|c|c|c|c|c|}
\hline \multicolumn{3}{|c|}{ Number of patients } & \multicolumn{3}{|c|}{ Age } & \multicolumn{3}{|c|}{ Effective diameter $(\mathrm{cm})$} & \multicolumn{3}{|c|}{ Scan length (mm) } \\
\hline 152 & 75 & 77 & $\begin{array}{l}52 \pm 18 \\
(18-89)\end{array}$ & $\begin{array}{l}53 \pm 18 \\
(18-89)\end{array}$ & $\begin{array}{l}51 \pm 18 \\
(18-89)\end{array}$ & $\begin{array}{l}28 \pm 2.8 \\
(19.5-36.3)\end{array}$ & $\begin{array}{l}27 \pm 2.6 \\
(20.0-33.3)\end{array}$ & $\begin{array}{l}28 \pm 3.0 \\
(19.5-36.3)\end{array}$ & $\begin{array}{l}296 \pm 33 \\
(219-390)\end{array}$ & $\begin{array}{l}312 \pm 32 \\
(240-390)\end{array}$ & $\begin{array}{l}280 \pm 24 \\
(219-336)\end{array}$ \\
\hline
\end{tabular}

Table 2. The effective tube current-time product, $\mathrm{CTDI}_{\mathrm{VOL}}$ and DLP values used for CTPA examination.

\begin{tabular}{|c|c|c|c|c|c|c|c|c|}
\hline \multicolumn{3}{|c|}{ Tube current-time product (mAs) } & \multicolumn{3}{|c|}{$\mathrm{CTDI}_{\mathrm{VOL}}(\mathrm{mGy})$} & \multicolumn{3}{|c|}{ DLP $(\mathrm{mGy} \mathrm{cm})$} \\
\hline $\begin{array}{l}51.9 \pm 15.6 \\
(30-100)\end{array}$ & $\begin{array}{l}52.5 \pm 14.7 \\
(30-85)\end{array}$ & $\begin{array}{l}60.5 \pm 15.5 \\
(30-100)\end{array}$ & $\begin{array}{l}4.6 \pm 1.4 \\
(2.4-11.5)\end{array}$ & $\begin{array}{l}4.2 \pm 1.2 \\
(2.5-6.8)\end{array}$ & $\begin{array}{l}5.0 \pm 1.6 \\
(2.4-11.5)\end{array}$ & $\begin{array}{l}139.8 \pm 42.3 \\
(12.1-353.9)\end{array}$ & $\begin{array}{l}136.7 \pm 35.6 \\
(73.3-224.9)\end{array}$ & $\begin{array}{l}142.8 \pm 48.1 \\
(12.1-353.9)\end{array}$ \\
\hline
\end{tabular}

Table 3. The effective dose values (mSv) calculated for CTPA examinations.

\begin{tabular}{|c|c|c|c|c|c|c|c|c|c|c|c|}
\hline \multicolumn{12}{|c|}{ Effective dose values (mSv) } \\
\hline Mean & SD & Maximum & Minimum & Mean & $\mathrm{SD}$ & Maximum & Minimum & Mean & $\mathrm{SD}$ & Maximum & Minimum \\
\hline 2.04 & 0.61 & 4.1 & 0.92 & 1.61 & 0.33 & 2.49 & 0.92 & 2.46 & 0.53 & 4.1 & 1.41 \\
\hline
\end{tabular}

risk estimation. For the REID estimation of a given patient in the PCXMC, data include the 'Age', 'Gender' and mortality "Statistics" (Euro-American) of the patient were selected in the program. A more thorough explanation of the PCXMC program and its calculation method can be found in the technical program document (Tapiovaara and Siiskonen, 2008).

\section{Results}

The characteristics of patients include number, age, effective diameter and length of the scanning area are shown in Table 1 for both genders separately. In this study, CTPA examinations were equally performed among female (77/152, $51 \%)$ and male $(75 / 152,49 \%)$ patients.

Maximum, minimum, mean, and standard deviation of the effective tube current-time product (mAs), $\mathrm{CTDI}_{\mathrm{VOL}}$, and DLP values are separately shown in Table 2 for all patients and for male and female patients separately.

Table 3 shows maximum, minimum, mean, and standard deviation of the effective dose values for all patients. The effective dose was much higher in females $(2.46 \mathrm{mSv})$ than in males $(1.61 \mathrm{mSv})$. This is explained by the fact that the effective dose depends not only on the scanning parameters but also on the radiosensitivity of the tissues irradiated. In particular, breast is highly radiosensitive in young females.

Mean and standard deviation of organ dose values from CTPA scans are summarized in Table 4. Directly irradiated organs, like the lung, heart, thymus and esophagus, received doses of 5.98, 5.96, 6.2 and $4.52 \mathrm{mGy}$, respectively. For female patients, breast doses were relatively high at $5.53 \mathrm{mGy}$. Doses to thyroid, liver, stomach, adrenal, pancreas and spleen positioned in the proximity of the scan range were 2.00, 2.77, $2.00,3.96,2.66$, and $2.45 \mathrm{mGy}$, respectively.
Table 4. Organ dose values (mGy) obtained in CTPA examinations.

\begin{tabular}{llllll}
\hline Organ or tissue & Mean & SD & Organ or tissue & Mean & SD \\
\hline Bladder & 0.01 & 0.01 & Adrenal & 3.96 & 1.31 \\
Brain $^{1}$ & 0.04 & 0.02 & Extra thoracic area & 0.54 & 0.22 \\
Breast $^{1}$ & 5.53 & 1.34 & Gall bladder & 0.90 & 0.54 \\
Colon $^{\text {Esophagus }}$ & 0.09 & 0.06 & Heart & 5.96 & 1.21 \\
Gonads $^{2}$ & 4.52 & 0.87 & Kidneys & 0.98 & 0.71 \\
Gonads $^{1}$ & 0.00 & 0.00 & Lymphatic node & 1.39 & 0.31 \\
Liver $^{\text {Lung }}$ & 0.04 & 0.02 & Muscle & 1.39 & 0.31 \\
Red bone marrow $^{2.77}$ & 1.01 & Oral mucosa & 0.20 & 0.11 \\
Salivary glands & 0.55 & 0.12 & Prostate & \\
Skeleton $^{2}$ & 0.09 & Small intestine & 0.11 & 0.08 \\
Skin & 3.85 & 0.86 & Spleen & 2.45 & 1.09 \\
Stomach & 1.30 & 0.36 & Thymus & 6.91 & 1.33 \\
Thyroid & 2.01 & 0.87 & Uterus & 0.00 \\
\hline
\end{tabular}

1 For female.

2 For male.

Each graph in Figures 1 and 2 shows results of the REID (per million) due to CTPA scans for male and female patients as a function of age. The REID values were considerably higher in females than in males. In both genders, the radiation risk decreased with patient age, with the decrease being much steeper for women. The REID value for a 20 -year-old woman was 1 in 4032 (248 per million). For a 40-year-old woman, the REID value was 1 in 5025 (199 per million); for a 60-year-old, the REID value was 1 in 6536 (153 per million); and for an 80 -year-old, the REID value was 1 in 13,513 (74 per million). 


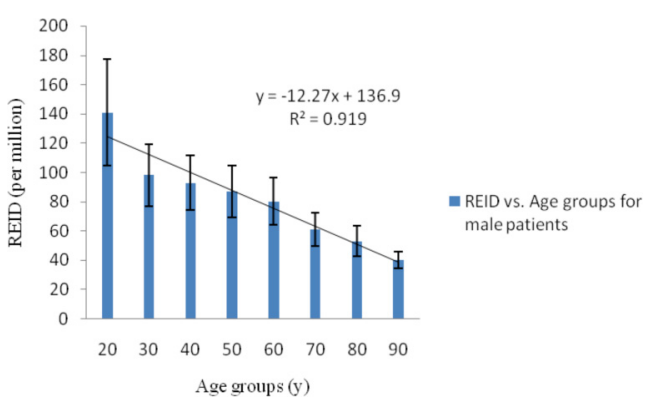

Fig. 1. The results of the REID (per million) due to CTPA scans for male patients as a function of age, with error bars showing the corresponding standard deviations.

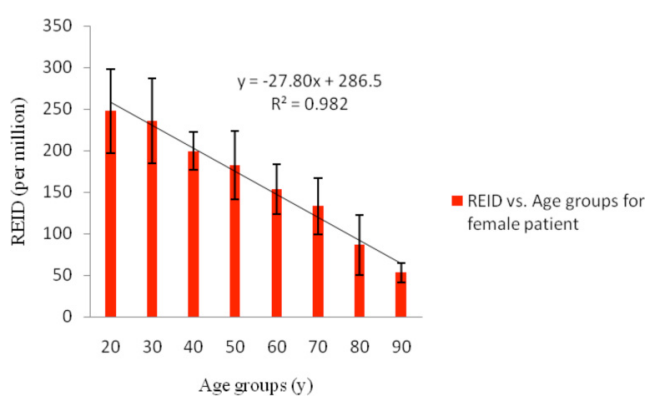

Fig. 2. The results of the REID (per million) due to CTPA scans for female patients as a function of age, with error bars showing the corresponding standard deviations.

For a 20-year-old man, the REID value was 1 in 7092 (141 per million), equivalent to the risk to a 66-year-old woman. Risks to men also decreased as a function of age, to 1 in 10,753 ( 93 per million) for a 40-year-old, 1 in 12,453 (80 per million) for a 60-year-old, and 1 in 18,867 (53 per million) for an 80 -year-old.

\section{Discussion}

The major findings of the current study are as follows: (1) the mean effective dose for a CTPA scan was $1.61 \pm 0.33 \mathrm{mSv}$ in men and $2.46 \pm 0.53 \mathrm{mSv}$ in women. (2) The REID values ranged from $248 / 1000,000$ for a 20 -year-old female patient to $53 / 1000,000$ for an 80 -year-old male patient.

The above-mentioned effective doses were comparable to those reported in Heyer et al. (2007) study (1.37-2.44 mSv) and Yilmaz et al. (2013) (1.2-4.7 mSv). However, the effective doses computed by Woo et al. (2012) $(8.4 \mathrm{mSv}$ for females and $9.7 \mathrm{mSv}$ for males) were higher than those calculated in our study. Higher effective dose values in Woo et al. (2012) study may be due to the higher exposure factors $(120 \mathrm{kVp}$ and $100-250 \mathrm{~mA}$ ) in that study compared with those in other investigations. Another reason for this difference is that Woo et al. (2012) calculated the effective dose by multiplying the total DLP by the chest-specific conversion coefficient of $0.017 \mathrm{mSv} \mathrm{mGy}^{-1} \mathrm{~cm}^{-1}$ determined from Monte Carlo simulations of chest CT (European Commission, 1996). Because calculations based on DLP reduces the effective dose estimates for women and increases it for men in comparison to ImpactDose estimates, this method is not appropriate. Also, the mentioned chest-specific conversion coefficient is a composite value for female and male patients, thereby underestimating breast dose in women but overestimating it in men (Einstein et al., 2008).

Comparison of the REID values of this survey with other studies was difficult. Because to date, only one study (Woo et al., 2012) has estimated the REID values for CTPA. As shown in Figures 1 and 2, the REID values were markedly higher in females (53-248 per million) than in males (40-141 per million). This is consistent with the results of Woo et al. (2012) who also reported higher radiation risks for women, but the magnitude of radiation risks in their results were higher than those estimated in the present study. This may also be attributed to the use of different methodology and higher exposure factors in that study.

Effects of age at exposure and attained age are important modifiers of the radiation risks and BEIR VII (NRC, 2006) reported an association between age at exposure and/or attained age and the risk of radiation induced cancer. In our study, also the radiation risk increased with decreasing age at time of exposure for both genders. This is attributed to the inclusion of radiosensitive organs such as the female breast, thyroid gland, and lung in the scan field. These organs in young adults are highly radiosensitive; their radiosensitivity drop abruptly with age (NRC, 2006).

Our study had several limitations. First, the cancer risk models, which were based on the linear no-threshold (LNT) model for low-dose radiation risk, have inherent uncertainty. However, most surveys (Pierce and Preston 2000; Cardis et al., 2007) agree that the LNT model is a reasonable assumption for low-dose radiation risk estimation. Second, another important aspect that has to be considered is limitation in the epidemiologic data that contributes to the uncertainty of the cancer risk estimation. For assessment of REID values, PCXMC uses the Euro-American mortality data which are available from ICRP Publication 103 (2007). These data do not necessarily correspond to the average mortality statistics of all countries in the world, but fortunately Iranian population has similar mortality data with Euro-American population (Vatandoost et al., 2016). Third, calculation methods for organ doses were based on the mathematical phantoms (ORNL series). Although several aspects in this study were matched to those in individual patient scans, including age, gender, effective diameter of patients, scan length, effective tube current-time product, tube voltage, scan range, gantry rotation time, pitch, CTDI and DLP values, it was not possible to precisely simulate all aspects of patient anatomy. For further investigations, we suggest to use the Monte Carlo-based simulation program ImpactMC (Deak et al., 2008) for dose calculations. With this program, the organ doses are calculated from the CT images obtained for each patient; therefore, dose calculations are specific to patient anatomy.

\section{Conclusion}

This study was set out to determine the REID values associated with CTPA. The REID values varied from 53 to 248 per million depending on the age and gender of patients. The REID values were considerably higher for females than males, 
and higher for younger compared to older patients. An approach for minimizing the radiation risk is to reduce the number of CT examinations. Awareness of referring physicians about the REID values obtained in this study will help them to better evaluate radiation exposure risk, before ordering a CTPA examination. Also, the REID values can be comprehensible and easily understandable for referring physicians and radiologists to justify each CTPA examination in order to reduce unnecessary examinations or use alternative imaging modalities with lower risk. Other alternative modalities for imaging of the pulmonary arteries and diagnosis of PE are magnetic resonance angiography, Doppler ultrasound, Ventilation-perfusion (V/Q) scanning and each has its advantages and disadvantages. For example, while CTPA is regarded as a highly sensitive and specific test for PE (Fedullo and Tapson, 2003), the total effective dose from CTPA is about 5 times greater than that from V/Q scans (Jones et al., 2012).

\section{References}

AAPM. 2011. Size-Specific Dose Estimates (SSDE) in Pediatric and Adult Body CT Examinations. Report of AAPM Task Group 204. College Park, Md: American Association of Physicists in Medicine, 14: 14-15.

Balonov M, Shrimpton P. 2012. Effective dose and risks from medical X-ray procedures. Ann. ICRP 41(3): 129-141.

Brenner DJ. 2008. Effective dose: a flawed concept that could and should be replaced. Br. J. Radiol. 81: 521-523.

Cardis E, Vrijheid M, Blettner M, et al. 2007. The 15-country collaborative study of cancer risk among radiation workers in the nuclear industry: estimates of radiation-related cancer risks. Radiat. Res. 167(4): 396-416.

Chen W, Kolditz D, Beister M, Bohle R, Kalender WA. 2012. Fast onsite Monte Carlo tool for dose calculations in CT applications. Med. Phys. 39(6): 2985-2996.

Costantino MM, Randall G, Gosselin M, Brandt M, Spinning K, Vegas CD. 2008. CT angiography in the evaluation of acute pulmonary embolus. Am. J. Roentgenol. 191(2): 471-474.

Cristy M, Eckerman K. 1987. Specific absorbed fractions of energy at various ages from internal photon sources. ORNL/TM-8381. Oak Ridge, TN: Oak Ridge National Laboratory.

Deak P, Van Straten M, Shrimpton PC, Zankl M, Kalender WA. 2008. Validation of a Monte Carlo tool for patient-specific dose simulations in multi-slice computed tomography. Eur. Radiol. 18(4): 759-772.

Diederich S. 2003. Radiation dose in helical CT for detection of pulmonary embolism. Eur. Radiol. 13(7): 1491-1493.

Einstein AJ, Sanz J, Dellegrottaglie S, et al. 2008. Radiation dose and cancer risk estimates in 16-slice computed tomography coronary angiography. J. Nucl. Cardiol. 15(2): 232-240.

European Union, European Commission, Directorate-General XIIScience, R. and Development. 1996. European guidelines on quality criteria for diagnostic radiographic images in paediatrics. Office for Official Publications of the European Communities.

Fedullo PF, Tapson VF. 2003. The evaluation of suspected pulmonary embolism. N. Engl. J. Med. 349(13): 1247-1256.

He J, Wang F, Dai HJ, et al. 2012. Chinese multi-center study of lung scintigraphy and CT pulmonary angiography for the diagnosis of pulmonary embolism. Int. J. Cardiovasc. Imag. 28(7): 1799-1805.

Heyer CM, Mohr PS, Lemburg SP, Peters SA, Nicolas V. 2007. Image quality and radiation exposure at pulmonary $\mathrm{CT}$ angiography with 100-or 120-kvp protocol: prospective randomized study. Radiology 245(2): $577-83$.
Huda W, He W. 2012. Estimating cancer risks to adults undergoing body CT examinations. Radiat. Prot. Dosim. 150(2): 168-179.

IAEA. 2012. Quality assurance programme for computed tomography: Diagnostic and therapy applications. Vienna: Human Health Series No. 19.

ICRP Publication 34. 1982. Protection of the Patient in Diagnostic Radiology. Ann. ICRP 9: 2-3.

ICRP Publication 60. 1991. 1990 Recommendations of the International Commission on Radiological Protection. Oxford: Annals of the ICRP, Pergamon Press.

ICRP Publication 73. 1996. Radiological protection and safety in medicine. Ann. ICRP 26: 2-3.

ICRP Publication 26. 1977. 1977 recommendations of the International Commission on Radiological Protection. Ann. ICRP 1: 3 .

ICRP Publication 103. 2007. The 2007 recommendations of the international commission on radiological protection. Ann. ICRP 37: $1-332$.

ICRP Publication 110. 2009. Adult reference computational phantoms. Ann. ICRP 39: 1-164.

Jones JG, Mills CN, Mogensen MA, Lee CI. 2012. Radiation dose from medical imaging: a primer for emergency physicians. West $J$. Emerg. Med. 13(2): 202-210.

Kalender WA, Schmidt B, Zankl M, Schmidt M. 1999. A PC program for estimating organ dose and effective dose values in computed tomography. Eur. Radiol. 9(3): 555-562.

Li X, Samei E, Segars WP, Sturgeon GM, Colsher JG, Frush DP. 2011. Patient-specific radiation dose and cancer risk for pediatric chest CT. Radiology 259(3): 862-874.

Martin CJ. 2007. Effective dose: How should it be applied to medical exposures? Br. J. Radiol. 80(956): 639-647.

Martin CJ. 2011. Effective dose: practice, purpose and pitfalls for nuclear medicine. J. Radiol. Prot. 31(2): 205.

McCollough CH, Christner JA, Kofler JM. 2010. How effective is effective dose as a predictor of radiation risk? Am. J. Roentgenol. 194(4): 890-896.

Musset D, Parent F, Meyer G, et al. 2002. Diagnostic strategy for patients with suspected pulmonary embolism: a prospective multicentre outcome study. Lancet 360(9349): 1914-1920.

NRC 2006. Committee to assess health risks from exposure to low levels of ionizing radiation, national research council (NRC). Health risks from exposure to low levels of ionizing radiation: BEIR VII - Phase 2. Washington, DC: National Academy of Sciences.

O'Neill JM, Wright L, Murchison JT. 2004. Helical CTPA in the investigation of pulmonary embolism: a 6-year review. Clin. Radiol. 59(9): 819-825.

O'Neill J, Murchison JT, Wright L, Williams J. 2005. Effect of the introduction of helical CT on radiation dose in the investigation of pulmonary embolism. Br. J. Radiol. 78: 46-50.

Pierce DA, Preston DL. 2000. Radiation-related cancer risks at low doses among atomic bomb survivors. Radiat. Res. 154(2): 178-186.

Pradhan AS, Kim JL, Lee JI. 2012. On the use of "effective dose" (E) in medical exposures. J. Med. Phys. 37(2): 63.

Preston DL, Ron E, Tokuoka S, et al. 2007. Solid cancer incidence in atomic bomb survivors: 1958-1998. Radiat. Res. 168(1): 1-64.

Sahbaee P, Segars WP, Samei E. 2014. Patient-based estimation of organ dose for a population of 58 adult patients across 13 protocol categories. Med. Phys. 41(7): 072104.

Shope TB, Gagne RM, Johnson GC. 1981. A method for describing the doses delivered by transmission X-ray computed tomography. Med. Phys. 8(4): 488-495. 
Surgeon General 2008. Acting Surgeon General issues call to action to prevent deep vein thrombosis and pulmonary embolism. Available at: http://www.surgeongeneral.gov/news/pressreleases/ pr20080915.html.

Tapiovaara M, Siiskonen T. 2008. A Monte Carlo program for calculating patient doses in medical X-ray examinations, STUKA231.Helsinki: Radiation and Nuclear Safety Authority.

Turner AC, Zankl M, DeMarco JJ, et al. 2010. The feasibility of a scanner-independent technique to estimate organ dose from MDCT scans: using CTDIvol to account for differences between scanners. Med. Phys. 37(4): 1816-1825.

Turner AC, Zhang D, Khatonabadi M, et al. 2011. The feasibility of patient size-corrected, scanner-independent organ dose estimates for abdominal CT exams. Med. Phys. 38(2): 820-829.
Vatandoost N, Salehi AR, Kazemi M, et al. 2016. Genetic polymorphism of 8 Y-STR loci in native population of Isfahan province in central part of Iran, Ann. Hum. Biol. 44(2): 175-179.

Wells PS, Anderson DR, Rodger M, et al. 2001. Excluding pulmonary embolism at the bedside without diagnostic imaging: management of patients with suspected pulmonary embolism presenting to the emergency department by using a simple clinical model and d-dimer. Ann. Intern. Med. 135(2): 98-107.

Woo JK, Chiu RY, Thakur Y, Mayo JR. 2012. Risk-benefit analysis of pulmonary CT angiography in patients with suspected pulmonary embolus. Am. J. Roentgenol. 198(6): 1332-1339.

Yilmaz Ö, Üstün ED, Kayan M, et al. 2013. Diagnostic quality of CT pulmonary angiography in pulmonary thromboembolism: a comparison of three different kV values. Med. Sci. Monit. 19: 908.

Cite this article as: Karimizarchi H, Chaparian A. 2017. Estimating risk of exposure induced cancer death in patients undergoing computed tomography pulmonary angiography. Radioprotection 52(2): $81-86$ 